\title{
PERFORMANCE EVALUATION OF VARIOUS TYPES OF NODES IN MANET WITH DSR ROUTING PROTOCOLS
}

\author{
Puneet Mittal ${ }^{1}$, Paramjeet Singh ${ }^{2}$, Shaveta Rani ${ }^{3}$ \\ ${ }^{I}$ Dept. of Computer Engg, Govt. Polytechnic College, Bathinda, Punjab, India \\ ${ }^{2,3}$ Dept. of Computer Science and Engg, GZS PTU Campus, Bathinda, Punjab, India \\ engg.puneet@yahoo.co.in,param2009@yahoo.com,garg_shavy@yahoo.com
}

\begin{abstract}
Day by day, wireless communication popularity growing fast, it has led wireless communication data rates higher and made its prices cheaper, that's why wireless communication is growing so fast. The latest technological demands now a day's arising from laptops, wireless devices such as wireless local area networks (LANs) etc. In this paper we compared different types of node in MANET like static nodes, dynamic nodes and dynamic nodes with trajectory by using Dynamic routing protocol (DSR) and compared the performance of these nodes by using OPNET simulator 14.5. The performance is evaluated under different parameters like Data Dropped (Retry Threshold Exceeded), Load, Media access delay and Network Load by using FTP load.
\end{abstract}

Keywords: MANET, Peak Value, Protocol, Trajectory.

\section{INTRODUCTION}

In mobile ad-hoc network (MANET) is a collection of mobile nodes and any node can disappear or join anytime at any location in the network. Manet has multi-hop commutation capability. There is no centralized administration and no dedicated routers or other network infrastructures to support the nodes. Any node can be a sender or receiver as well as a router and they can forward the data packets to another node. In these types of networks each node works as an independent router. MANET is a fast growing area of research [1].

A Wireless Network transmits data from node to node by using a central base station (Access Point). There are two types of wireless network: (1) Standalone Architecture in which all devices are directly communicating with each other in peer to peer communication node. (2) Centrally Coordinated Architecture in which all devices are connected with the help of access point. MANET is one of the types of Ad hoc network in which of wireless nodes (connected by wireless links) that performing a dynamically network without using any established infrastructure or centralized administration. It can be setup anywhere and anytime with free to move randomly and act as router.

In MANET, protocols are classification into three categories: (1) Proactive protocols or table driven provide fast response to topology changes by continuously monitoring topology changes and disseminating the related information as needed over the network [2] like Optimized Link State Routing (OLSR), Destination-Sequenced Distance-Vector (DSDV) Routing Algorithm, Wireless Routing Protocol (WRP), Global
State Routing (GSR), Fisheye State Routing (FSR), Hierarchical State Routing (HSR), Zone-based Hierarchical Link State Routing Protocol (ZHLS). (2) Reactive routing or on demand protocols such as Ad hoc in demand distance vector (AODV), find the route only when there is data to be transmitted as a result, generate low control traffic and routing overhead. Dynamic Source routing protocol (DSR), each data packet contains complete routing information to reach its dissemination and each node uses caching technology to maintain route information. (3) Hybrid protocol could be derived from the two previous ones, containing the advantages of both the protocols.

Among all on-demand routing protocols DSR is straight forward, simple, and recently a lot of modifications and improvements are going on in this protocol. Some are introducing security and power issues and many other concepts. Therefore we are closely examining the protocol and its performance under various working conditions using simulation techniques. This paper is organized as follows. In sec. 2, we describe the Dynamic routing protocol (DSR) in MANET. Sec 3, gives various parameters traffic loads in MANET. In sec 4, simulation environment in OPNET SIMULATOR 14.5 is given. Sec 5 shows the results and discussion about the performance of various nodes in MANET by using DSR protocol. Conclusion is given in Sec 6 .

\section{DYNAMIC ROUTING PROTOCOLS IN MANET}

DSR is also a reactive routing protocol. It uses the concept of source routing [3]. In source routing the sender knows all hopby-hop routes to the destination. All the routes are stored in 
the route cache. When a node attempts to send a data packet to a destination it does not know the route. In DSR each node maintains a route cache with route entries which are continuously updated. The advantage of DRS is that no periodic routing packets are required. It is used to updates its route caches by finding new routes [4]. DSR has also the capability to handle unidirectional links. The sender of the packets selects and controls the route used for its own packets, which also supports features such as load balancing. All routes used are guaranteed to be free of loops as the sender can avoid duplicate hops in the selected routes. The following sections introduce state machines that implement a simple DSR routing protocol without caching. There are basically 4 separate state machines to implement that each handles one of the following events [5]:

1. SENDING DATA: When a node wants to send data and data message is triggered as a send request then the next hop has be determined. A route request has to be broadcast to discover a route to the destination node. After receiving the route reply from the destination node the actual data message can be transmitted via the newly discovered.

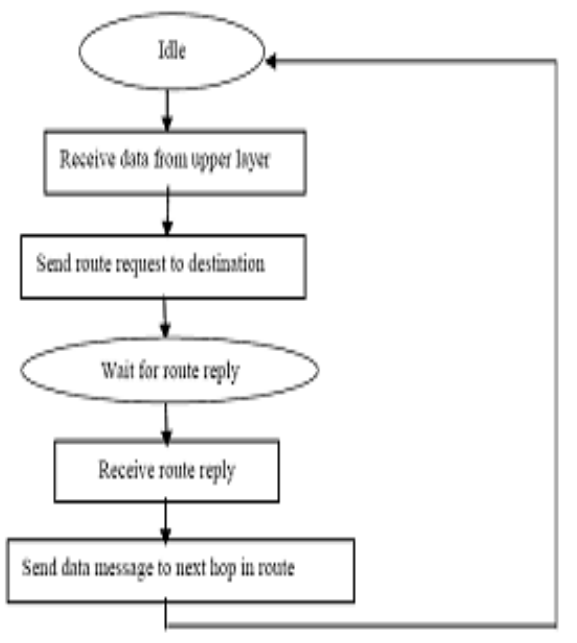

Fig 1: Flow Chart Sending Activity Diagram

2. INCOMING ROUTE REQUEST MESSAGE: When a route request message is received by a node, several tasks have to be done depending on the content of the received message. Firstly, it is checked whether the message was already processed earlier by this node. If yes, the request is simply discarded and no action is taken. If the route request is addressed to the receiving node, a route reply message has to be created and replied to the request's sender. In all other cases the route request's node list is extended by the own node ID and broadcasted to all neighboring nodes.

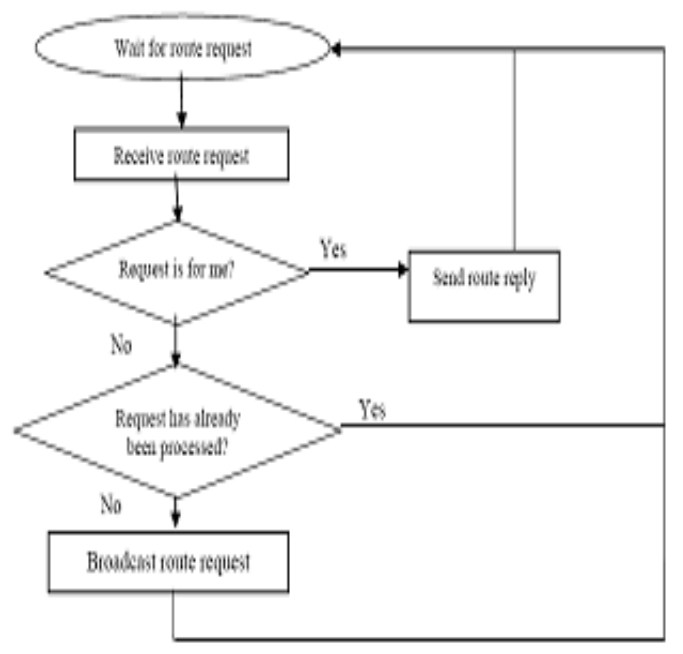

Fig 2: Flow Chart of Route Request

3. INCOMING ROUTE REPLY MESSAGE: In case a route reply message is received there are two cases: The receiving node is the destination of the message or it is an intermediate node on the route between sender and destination. If it is the destination node the data transmission that caused the route discovery can be accomplished. In case the route reply is received by an intermediate node, it is forwarded to the next node in the reply's node list.

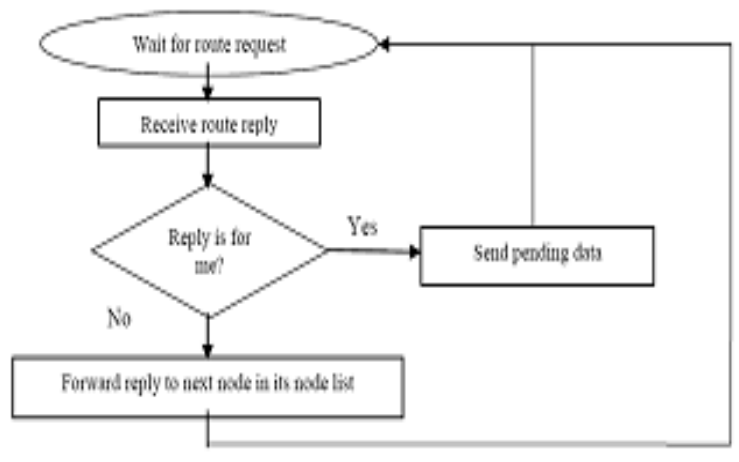

Fig 3: Flow Chart of Route Reply

4. INCOMING DATA MESSAGE: If the data message is addressed to the receiving node (it is the last hop of the route) the payload can be handed to the upper layer to be processed by any application. The routing mechanism has finished. If not, the next hop is determined from the data message's node list and the packet is forwarded to this node. 


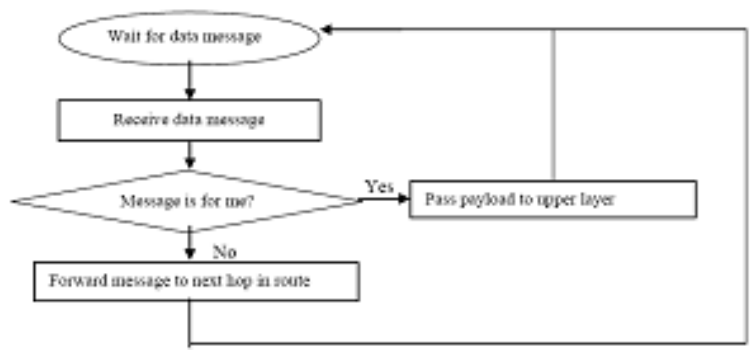

Fig 4: Flow Chart of Data Message

DSR contains 2 phases:

\subsection{Route Discovery (Find A Path)}

If sender node has in his route cache a route to the destination node, this route is immediately used. If not, the route discovery protocol is started:

Step 1: sender node sends a route request packet by flooding the network. Each route request packet contains: route record, initiator address, request ID

Step 2: if the route discovery is successful the initiating host receives a route reply packet.

Step 3: when any host receives a route request packet, it processes the request accounting to the following steps.

a) If <initiator address, request id> is found I this host then discards the route request packet.

b) If this host's address is already listed in the route record discard the route request packet.

c) If the target of the request matches this host's address return a copy of this route in a route reply packet to the initiator.

d) Otherwise, append this host's address to the route record and re-broadcast the request.
After getting the route reply the sender send the data to the destination.

\subsection{Route Maintenance}

In DSR every node is responsible for confirming that the next hop in the source route receives the packet. Also each packet is only forwarded once by a node (hop-by-hop routing). If a packet can't be received by a node, it is retransmitted up to some maximum number of times until a confirmation is received from the next hop. Only if retransmission results in a failure, a Route Error message is sent to the initiator that can remove that source route from its route cache. So the initiator can check his route cache for another route to the target. If there is no route in the cache, a route request packet is broadcasted.

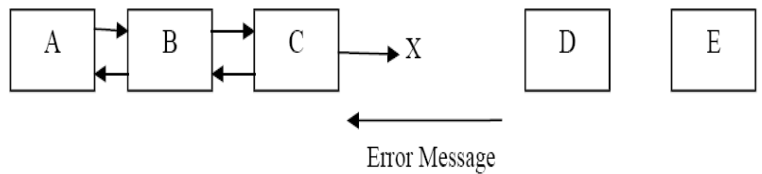

Fig 5: Example of DSR protocol

Step 1: if node $\mathrm{C}$ does not receive an acknowledgement form node D after some number of requests, it returns a Route Error to the initiator A.

Step 2: As soon as node receives the Route Error message, it deletes the broken-link-route from its cache. If A has another route to $\mathrm{E}$, it sends the packet immediately using this new route.

Step 3: Otherwise the initiator A is starting the Route Discovery process again.

\section{VARIOUS PARAMETERS IN TRAFFIC LOADS}

Table 1 shows the various simulation parameters.

\begin{tabular}{|l|l|l|}
\hline $\begin{array}{l}\text { SR. } \\
\text { NO. }\end{array}$ & PARAMETERS & DESCRIPTION \\
\hline 1 & Delay(Sec) & $\begin{array}{l}\text { Represents the end to end delay of all the packets received by the wireless LAN MACs } \\
\text { of all WLAN nodes in the network and forwarded to the higher layer. }\end{array}$ \\
\hline 2 & Load (Bits/Sec) & $\begin{array}{l}\text { Represents the total load submitted to wireless LAN layers by all higher layers in all } \\
\text { WLAN nodes of the network.(Global) }\end{array}$ \\
\hline 3 & $\begin{array}{l}\text { Media Access } \\
\text { Delay (Sec) }\end{array}$ & $\begin{array}{l}\text { For each frame, this delay is calculated as the duration from the time when it is inserted } \\
\text { into the transmission queue, which is arrival time for higher layer data packets and } \\
\text { creation time for all other frames types, until the time when the frame is sent to the } \\
\text { physical layer for the first time. }\end{array}$ \\
\hline 4 & $\begin{array}{l}\text { Throughput } \\
\text { (Bits/Sec) }\end{array}$ & $\begin{array}{l}\text { Represents the total number of bits (in bit/sec) forwarded from wireless LAN layers to } \\
\text { higher layers in all WLAN nodes of the network. }\end{array}$ \\
\hline 5 & $\begin{array}{l}\text { Bits/sec) } \\
\text { higher layers in all WLAN nodes of } \\
\text { the network, and it is difficult for the network to handle all this traffic so it is called the } \\
\text { network load. The efficient network can easily cope with large traffic coming in, and to } \\
\text { make a best network, many techniques have been introduced.(node network). }\end{array}$ \\
\hline 6 & $\begin{array}{l}\text { Retransmission } \\
\text { (Packets) }\end{array}$ & $\begin{array}{l}\text { The number of times data has to be retransmitting i.e called Retransmission Attempts. } \\
\text { How many no of times data has to be retransmit by the Source node. }\end{array}$ \\
\hline
\end{tabular}


All these parameters help us to evaluate the best routing protocol between them. All the parameters that have taken play a very vital role to judge or evaluate the performance of the wireless network. In this paper we can use Data Dropped (Retry Threshold Exceeded), Load, Media access delay and Network Load Parameters to evaluate the performance of various nodes in Manet.

\section{SIMULATION ENVIROMENT}

Several researchers have done the qualitative and quantative analysis of ad hoc routing protocol by means of different performance metrics. They have used different simulators for this purpose which is one of several tools provided from the OPNET Technologies suite. For undertake the experimental evaluation, the most recently available version, namely OPNET MODELER 14.5 has been adopted in our study OPNET is one of the most extensively used commercial simulators based on Microsoft Windows Platform, which incorporates most of the MANET routing parameters compared to other commercial simulators available [6]. The network entities used during the design of the network model are wireless server, application configuration, profile configuration, mobility configuration and workstations (nodes).

Table 2: Simulation parameters

\begin{tabular}{|l|l|}
\hline $\begin{array}{l}\text { SIMULATION } \\
\text { PARAMETER }\end{array}$ & VALUE \\
\hline Simulator & OPNET MODELER 14.5 \\
\hline Area & $200 \mathrm{~m}$ \\
\hline Network Size & 10 nodes \\
\hline Protocol & DSR \\
\hline Mobility Model & Random Way Point \\
\hline Traffic Type & FTP \\
\hline Simulation Time & $10($ Min $)$ \\
\hline Address Mode & IPv4 \\
\hline Inter Repetition time & Exponential $(4)$ \\
\hline Route Expiry Time & $300 \mathrm{Sec}$ \\
\hline Buffer Expiry time & $30 \mathrm{Sec}$ \\
\hline
\end{tabular}

Fig 6: Environment Scenario of 10 Fixed Nodes

In this Scenario, mobile nodes are fixed and work as static nodes..

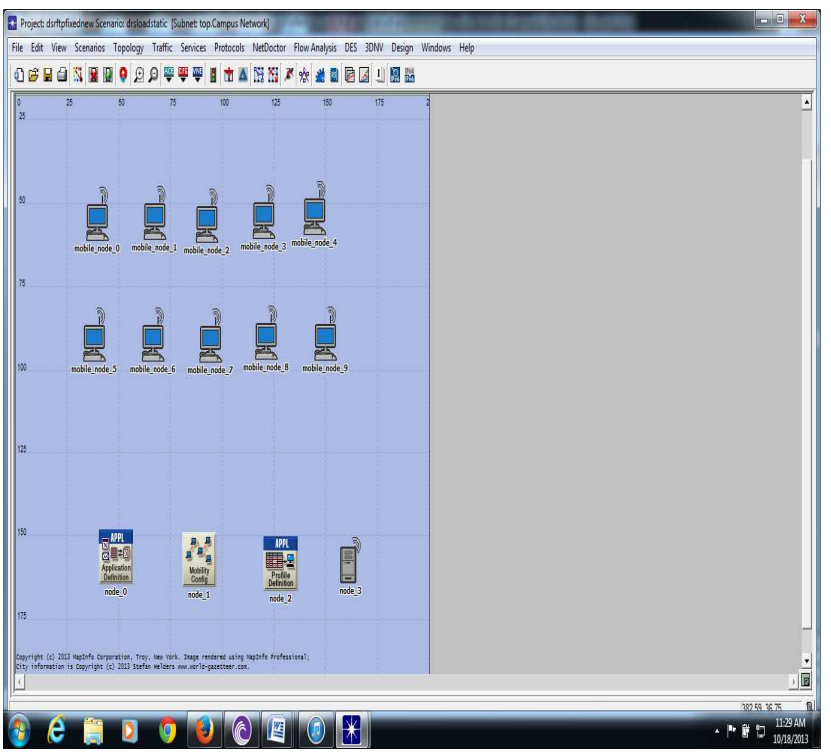

Fig 7: Environment Scenario of 10 Dynamic Mobile Nodes

Dynamic Mobile nodes are free to move arbitrarily which is typically multi-hop. It may change randomly and rapidly at unpredictable times. It consist of both bidirectional and unidirectional links.

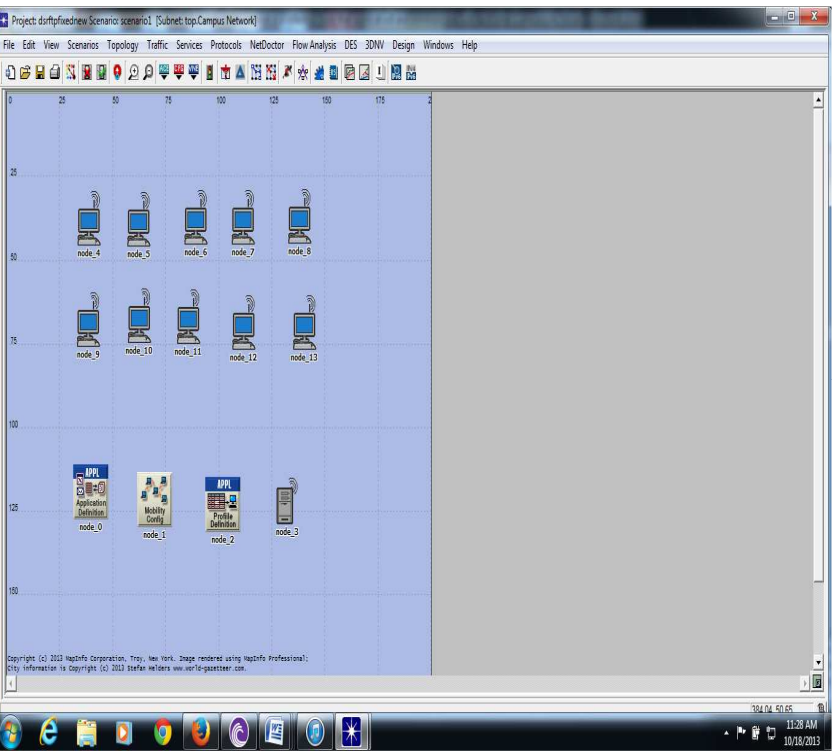




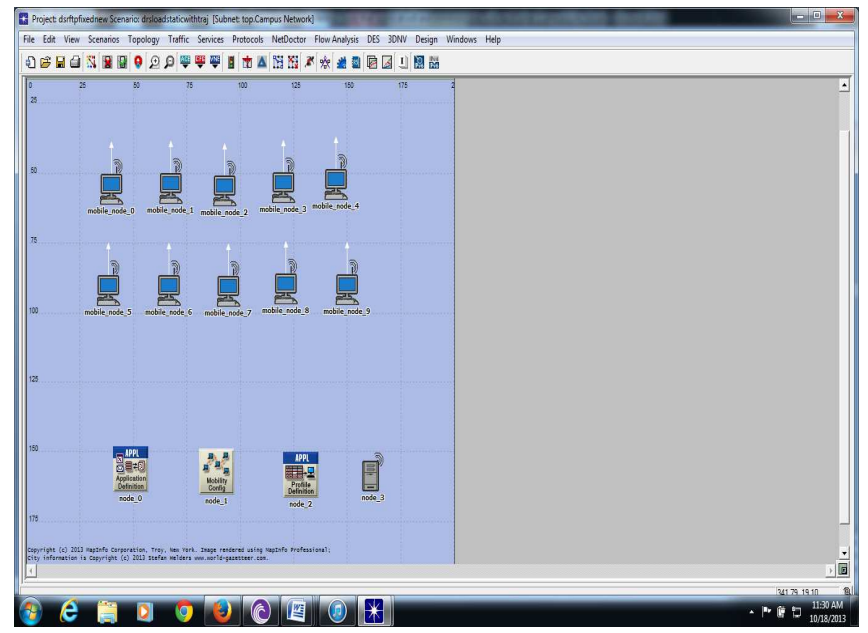

Fig 8: Environment Scenario of 10 Mobile Nodes with Vector Trajectory

A trajectory is defined as the path of a point moving with time. The trajectory obtained through visual tracking is important to many further analyses as they provide compact records of motion and characterize behaviour of each mobile. A key function of the vector system is the identification of mobile type. When the type is identified then this vector measure is compared with entries in a database and the best fit is determined to be the specific mobile type.

\section{RESULTS AND DISCUSSION}

\subsection{File Transfer Protocols (FTP) Load}

\subsubsection{Data Dropped (Retry Threshold Exceeded) (Bits/Sec)}
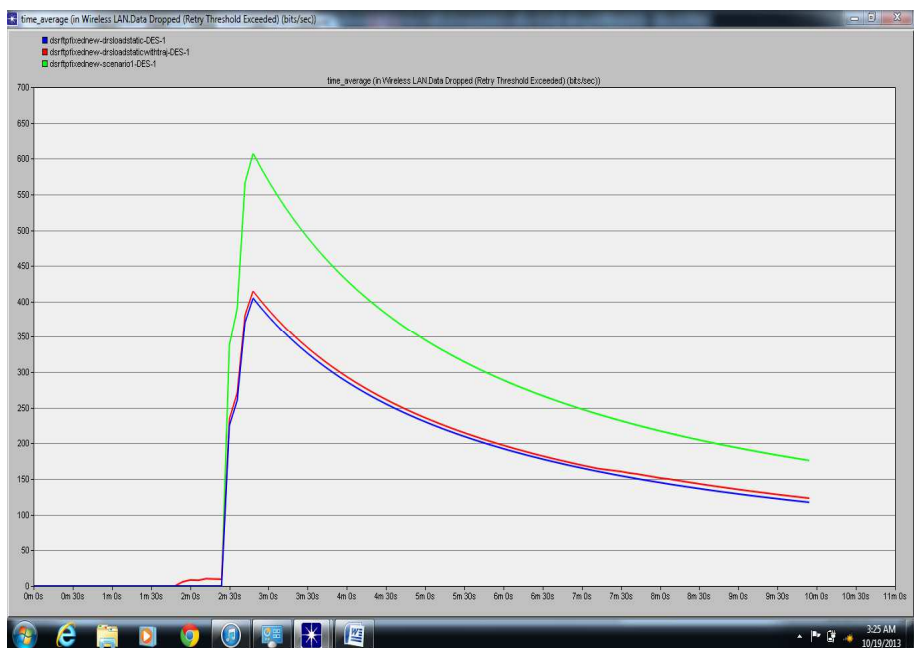

Fig 9: Comparison of DSR with static nodes, dynamic nodes and trajectory nodes for Data Dropped (Retry Threshold Exceeded)(Bits/Sec) in FTP Load
In figure 9, $\mathrm{X}$-axis denotes time which is in minutes and $\mathrm{Y}$ axis denotes data rate which is in bits/sec. It shows that the average peak value of Data Dropped (Retry Threshold Exceeded) is almost $606.896552 \mathrm{bits} / \mathrm{sec}$ for Static Nodes/ Fixed Nodes, $404.597701 \mathrm{bits} / \mathrm{sec}$ for Dynamic nodes and 414.528736 bits/sec for Dynamic nodes with Trajectory. After 10 minutes, it gradually drops as time progress and reaches to almost 176 bits/sec for Static Nodes / Fixed Nodes, 117.33333 bits/sec for Dynamic Nodes and $123.09333 \mathrm{bits} / \mathrm{sec}$ for Dynamic nodes with Trajectory.

\section{$5.2 \mathrm{Load}(\mathrm{Bit} / \mathrm{sec})$}

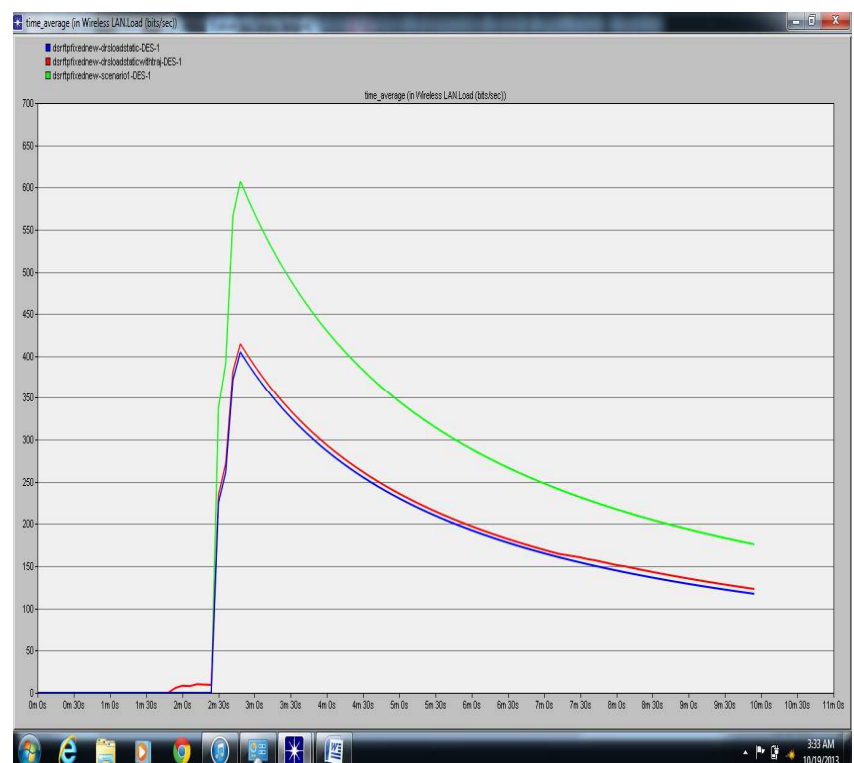

Fig 10: Comparison of DSR with static nodes, dynamic nodes and trajectory nodes for Load $(\mathrm{Bits} / \mathrm{Sec})$ in FTP Load

In figure $10, \mathrm{X}$-axis denotes time which is in minutes and $\mathrm{Y}$ axis denotes data rate which is in bits/sec. It shows that the average peak value of Load is almost $606.896552 \mathrm{bits} / \mathrm{sec}$ for Static Nodes/ Fixed Nodes, 404.597701 bits/sec for Dynamic nodes and $414.528736 \mathrm{bits} / \mathrm{sec}$ for Dynamic nodes with Trajectory. After 10 minutes, it gradually drops as time progress and reaches to almost $176 \mathrm{bits} / \mathrm{sec}$ for Static Nodes / Fixed Nodes, 117.33333 bits/sec for Dynamic Nodes and 123.09333 bits/sec for Dynamic nodes with Trajectory. 


\subsection{Media Access Delay (Sec)}

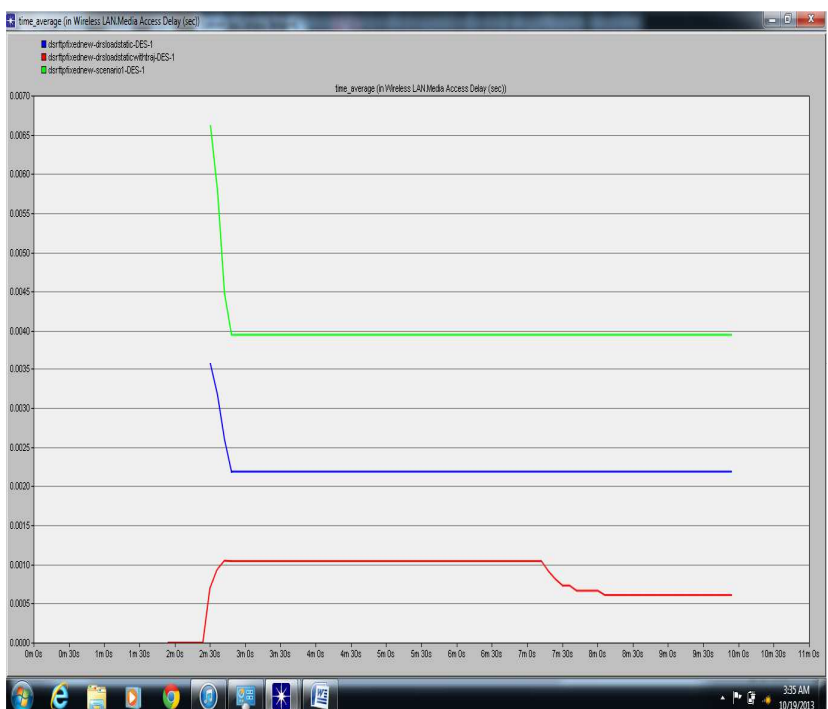

Fig 11: Comparison of DSR with static nodes, dynamic nodes and trajectory nodes for Media Access Delay (Sec) in FTP Load

In figure $11, \mathrm{X}$-axis denotes time in minutes and $\mathrm{Y}$-axis is denotes the time in seconds. It shows that the average peak value of Media access delay is almost 0.006626 seconds for Static Nodes/ Fixed Nodes, 0.003571 seconds for Dynamic nodes and 0.001047 seconds for Dynamic nodes with Trajectory. After 10 minutes, it gradually drops as time progress and reaches to almost 0.003949 seconds for Static Nodes / Fixed Nodes, 0.002187 seconds for Dynamic Nodes and 0.000608 seconds for Dynamic nodes with Trajectory.

\subsection{Network Load (bits/sec)}

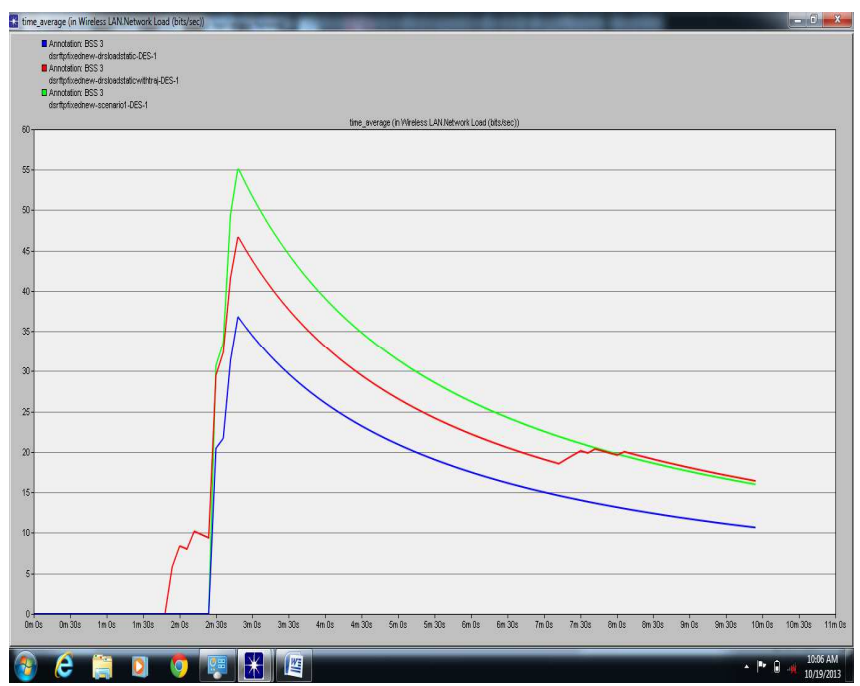

Fig 12: Comparison of DSR with static nodes, dynamic nodes and trajectory nodes for Network Load (Bits/Sec) in FTP Load

In figure $12, \mathrm{X}$-axis denotes time which is in minutes and $\mathrm{Y}$ axis denotes data rate which is in bits/sec. It shows that the average peak value of Network Load is almost 55.17241379 bits/sec for Static Nodes/ Fixed Nodes, 36.7816092 bits/sec for Dynamic nodes and $46.71264368 \mathrm{bits} / \mathrm{sec}$ for Dynamic nodes with Trajectory. After 10 minutes, it gradually drops as time progress and reaches to almost $16 \mathrm{bits} / \mathrm{sec}$ for Static Nodes / Fixed Nodes, $10.66667 \mathrm{bits} / \mathrm{sec}$ for Dynamic Nodes and 5.866667 bits/sec for Dynamic nodes with Trajectory.

Table 2 shows numeric values of various parameters taken into consideration for FTP load in Static nodes, Dynamic Nodes and Dynamic nodes with Trajectory in MANET by using Dynamic Routing Protocol. It gives the performance comparison of 3 Scenario: Static nodes, Dynamic Nodes and Dynamic nodes with Trajectory in MANET in terms of Data Dropped (Retry Threshold Exceeded), Load, Media access delay and Network Load for FTP load.

Table 3: Values of various parameters corresponding to 3 Scenario: Static nodes, Dynamic Nodes and Dynamic nodes with Trajectory in MANET

\begin{tabular}{|c|c|c|c|c|c|c|}
\hline \multirow{3}{*}{ Parameters } & \multicolumn{6}{|c|}{ DSR PROTOCOL } \\
\hline & \multicolumn{2}{|c|}{ Static Nodes } & \multicolumn{2}{|c|}{ Dynamic Nodes } & \multicolumn{2}{|c|}{$\begin{array}{lll}\text { Dynamic } & \text { Nodes } & \text { with } \\
\text { Trajectory } & & \\
\end{array}$} \\
\hline & Peak value & Drop value & Peak value & Drop value & Peak value & Drop value \\
\hline $\begin{array}{l}\text { Data dropped (retry threshold } \\
\text { exceeded) (bits } / \mathrm{sec})\end{array}$ & 606.89 & 176 & 404.59 & 117.33 & 414.52 & 123.09 \\
\hline Load (bits/sec) & 606.89 & 176 & 404.59 & 117.33 & 414.52 & 123.09 \\
\hline Media Access Delay $(\mathrm{Sec})$ & 0.0066 & 0.0039 & 0.0035 & 0.0021 & 0.0010 & 0.0006 \\
\hline Network Load (bits/sec) & 55.17 & 16 & 36.78 & 10.66 & 46.71 & 5.86 \\
\hline
\end{tabular}


As shown in Table 2, Static Nodes performs better than Dynamic Nodes and Dynamic Nodes with trajectory for Data dropped (Retry Threshold exceeded), Load, media Access Delay and Network Load. In overall Static Nodes performs better because it is easy to maintain and update the network than Dynamic nodes and dynamic nodes with Trajectory. In Static nodes network everything is done with the permission by network administration authority but in Dynamic every node is free to move or disappear/join. In dynamic network it is difficult to find any node location and also to maintain and update the network.

\section{CONCLUSIONS}

In this paper, we performed the comparison between three Scenarios: Static nodes, Dynamic Nodes and Dynamic nodes with Trajectory in MANET with traffic loads FTP in terms of Data dropped (Retry Threshold exceeded), Load, media Access Delay and Network Load. The results are taken in tabular form as well as graphical form by using OPNET Simulator 14.5. The results show that which Nodes performs better than another corresponding to FTP traffic load with DSR Protocol and some important parameters.

\section{REFERENCES}

[1]. Prasanna Padmanabhan, Le Gruenwald, Anita vallur, Mohammed Atiquzzaman, "A Survey of data replication techniques for mobile ad hoc network databases", The University of Oklahoma, School of Computer Science, Norman, OK 73019, USA, The VLDB Journal Springer (2008) 17:1143-1164.

[2]. Hui $\mathrm{Xu}$, Student Member, IEEE, Xianren Wu, Member, IEEE, Hamid R. Sadjapour, Senior Member, IEEE, and J.J. Garcai-Luna-Aceve, Fellow, IEEE, ACM, “ A Unified Analysis of Routing Protocls in MANETs". (2010)

[3]. Gurleen Kaur Walia and Charanjit Singh, "Node Density based performance Analysis of two Reactive Routing Protocols in Mobile Adhoc Networks", UCOE Department, Punjabi University, Patiala.(2011).

[4]. Parulpreet Singh, Ekta Barkhodia and Gurleen Kaur Wali, "Evaluation of various Traffic loads in MANET with DSR routing protocol through use of OPNET Simulator", Department of Electronics \& Communication, LPU, Phagwara Punjab, India. (May 2012).

[5]. Ad hoc and sensor networks, University of Paderborn, Computer Networks Group, 19 June 2007.

[6]. Zhi Ang Eu, NUS Graduate School for integrative sciences and Engineering, National University of Singapore and Hwee-Pink Tan, Winston K. G. Seah Networking Protocols Department, Institute for infocomm Research, A*STAR, "Routing and Relay Node Placement in Wireless Sensor Networks Powered by Ambient Energy Harvesting".

\section{BIOGRAPHIES:}

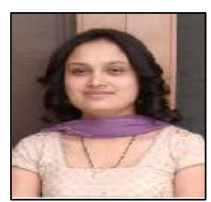

Puneet Mittal received B.Tech (CSE) form Punjab Technical University in 2007 and currently pursuing M.Tech (CSE) form Giani Zail Singh Punjab Technical University Campus, Bathinda. She is currently working as Lecturer Computer Engg Department, Govt. Polytechnic College, Bathinda. Her research area is Adhoc Networks routing protocols MANET.

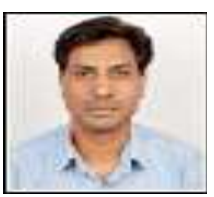

Paramjeet Singh reveived PhD from BITS, Pilani in 2009, M.S (Software System) form BITS, Pilani in 2002 and B.Tech (CSE) from Sant Longowal Institute of Engineering \& Technology, Longowal in $1998 . \quad$ He is currently working as Assistant Professor C.S.E Department, Giani Zail Singh Punjab Technical University Campus, Bathinda. His research area is Networking, Software Systems and Computer Graphics. He has to her contribution International Journals : 11, National Journals : 02, International Conerences : 12 and National Conferences : 30 .

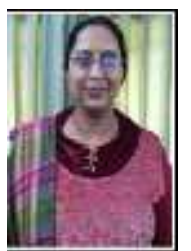

Shaveta Rani reveived PhD from BITS, Pilani in 2009, M.S (Software System) form BITS, Pilani in 2002 and B.Tech (CSE) from Sant Longowal Institute of Engineering \& Technology, Longowal in 1998. She is currently working as Assistant Professor C.S.E Department, Giani Zail Singh Punjab Technical University Campus, Bathinda. Her research area is Internetworking, Image Processing and Software Systems She has to her contribution International Journals : 11, National Journals : 02, International Conerences : 12 and National Conferences : 29. 